\title{
Research of Dimensional Accuracy about Shell Case Double Roller Spinning
}

\author{
Yu YANG \\ Changchun University of Science and Technology, Changchun, Jilin province, China \\ yangyu1983@sina.com
}

Keywords: Shifty Force, Power Spinning, Diametric Dimensional Accuracy, Surface Roughness.

\begin{abstract}
This essay proposed a spinning force calculation method based on rolling force, For the computing method of conventional spinning force sometimes has a great error by neglecting deposit metal before roller, and it is analyzed an effective and accurate way by testing a set of craft experiments. Then setting up a model on the basis of this method and conducting the stimulation test about shifty force of the projectile two rollers power spinning. The experimental results demonstrate that this experimental method can effectively increase projectile in its diametric dimensional accuracy and decrease its surface roughness on condition that projectile blank is No.20 steel; thickness is $10.6 \%$; the speed of main spindle is $50 \mathrm{r} / \mathrm{min}$; load is $3 \mathrm{~mm} / \mathrm{r}$.
\end{abstract}

\section{Introduction}

Spinning is a kind of chipless extrusion forming technology. The precision of the spinning products mainly comprises the parameters in two aspects: the precision of diameter of the spinning products and the surface roughness of the spinning products ${ }^{[1]}$. The precision of the spinning products is mainly affected by the feed rate; under the condition that other process parameters are kept constant, the increase of feed rate of spinning roller will cause rough surfaces of the spinning products. Otherwise, the reduction of the feed rate of spinning roller will not only lower the production rate, but cause the failure of profiling of the spinning products ${ }^{[2]}$. With a view to ensure the stable feed rate of the spinning roller, it is required to precisely determine the spinning force applied on the spinning roller in the process of spinning. At present, the evaluation of spinning force is conducted under the assumed condition that the metals are flowed axially in a stable and continuous manner; however, during actual spinning, the metal buildup caused due to the unsmooth flowing of metal will change the deformation rate of the spinning products and this is also the primary cause for the errors of being over $15 \%$ existed in the values of spinning force obtained respectively by the traditional algorithm and actual technical experiments ${ }^{[3]}$. Just because of the inaccuracy of the calculated values, the average value of spinning force is mostly applied for the setting of spinning force in the process of spinning; but this will cause the oversized feed amount in the earlier stage of spinning process and the undersized feed amount in the latter stage of the process and meanwhile the molding quality of the spinning products will become unsatisfactory. Although the dimensional accuracy of the spinning products will be improved by applying the traditional algorithm for the setting of spinning force and power spinning of changing spinning force, the relatively larger errors in calculated values will cause the corrugation frequently occurring in the process of spinning and then it will take lots of time to carry out the adjustment of process parameters and relevant experiments of spinning process ${ }^{[4]}$.

This paper has put forward a rolling force based algorithm to calculate spinning force on the basis of the analysis made of the changed reduction in wall thickness of 4 deformation zones of the spinning products in the process of spinning. The calculation of spinning force of various stages in the process of machining is conducted to analyze the effect of deformation of metal materials on the machining precision. Meanwhile, the Electrometric Method is applied to measure the radial, axial and tangential spinning force exerted on the spinning roller in the power spinning of projectile bodies. The experimental results have proved the accuracy of the algorithm. A simulation experiment on the power spinning of projectile bodies in which the varied spinning force is applied 
is conducted based on the algorithm. The experiments on 3 kinds of spinning processes are carried out, including the power spinning by adopting the spinning force of fixed value, the power spinning in which the varied spinning forces by traditional algorithm are applied and the power spinning in which the varied spinning forces by new algorithm are applied. The experimental results show that the dimensional accuracy of projectile body spun by power spinning in which the varied spinning forces are determined by new algorithm is more obviously improved when compared with that of the spinning products made by another two spinning processes. The result of the experiment is identical with that of simulation experiment.

\section{Principles of Spinning Process}

The spinning machine is mainly comprised of spindle box, spindle, mandrel, main lathe bed, side lathe bed, cross-shaped sliding table, spinning roller, tail jacking device, heating system, cooling system, hydraulic system and electric system. Working principle of metal spinning: mount and clamp the workblank on the mandrel and make it rotate together with the mandrel; meanwhile, the spinning roller is used to rotate around the workblank and relative feed is achieved between the spinning roller and mandrel in order to make the workblank to be pressed and produce continuous local plastic deformation; in this way, the hollow rotational parts can be obtained ${ }^{[5-6]}$.

Both of the reduction of surface smoothness caused by too much feed amount and failure of profiling of workblank caused by too less feed amount will affect the molding quality of spinning products. And the spinning products are mainly influenced in two aspects: dimensional tolerance of diameter and surface roughness.

To ensure a stable feed rate of spinning roller can effectively improve the shaping quality of spinning products and the prerequisite to achieve this is to accurately determine the spinning force applied on the spinning roller in the process of spinning. At present, the evaluation of spinning force is conducted under the assumed condition that the metals are flowed axially in a stable and continuous manner; and this only applies to the stable power spinning process. As a result, this paper put forward a rolling force based algorithm to calculate spinning force.

\section{Simulation Experiment}

The algorithm is used to build models and carry out simulation experiment of the power spinning in which double spinning rollers are equipped; and the experimental parameters obtained are identical with that given in Table 1. The spinning forces are applied on the spinning rollers in radial, axial and tangenial directions respectively, see Fig.1, Fig.2 and Fig.3 for details.

Tab.1 Experimental Parameters

\begin{tabular}{|c|c|c|}
\hline \multicolumn{2}{|c|}{ Parameters } & Numerical \\
\hline \multirow{3}{*}{ Blank } & Diameter & $100 \mathrm{~mm}$ \\
\cline { 2 - 3 } & Thickness & $20 \mathrm{~mm}$ \\
\cline { 2 - 3 } & Length & $100 \mathrm{~mm}$ \\
\cline { 2 - 3 } & Material science & 20 \\
\hline \multirow{3}{*}{ Rotary wheel } & Diameter & $200 \mathrm{~mm}$ \\
\cline { 2 - 3 } & Fillet radius & $10 \mathrm{~mm}$ \\
\cline { 2 - 3 } & Installation angle & $30^{\circ}$ \\
\hline \multirow{2}{*}{ Core film } & Diameter & $60 \mathrm{~mm}$ \\
\cline { 2 - 3 } & Length & $280 \mathrm{~mm}$ \\
\hline \multirow{2}{*}{ Feed } & Rev & $50 \mathrm{r} / \mathrm{min}$ \\
\cline { 2 - 3 } & Speed & $3 \mathrm{~mm} / \mathrm{r}$ \\
\cline { 2 - 3 } & Thinning & $10.6 \%$ \\
\hline
\end{tabular}




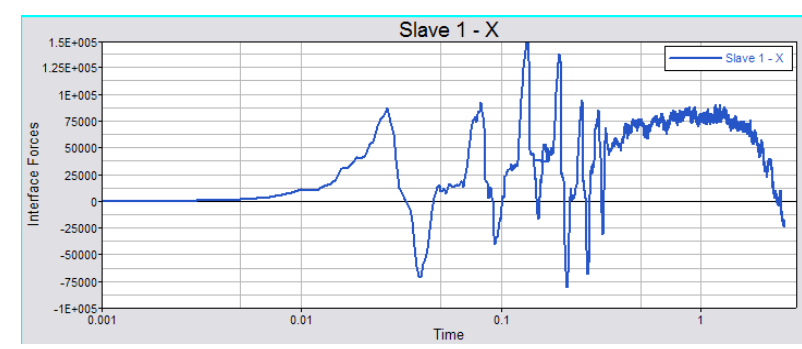

Fig.1 Radial Spinning Force

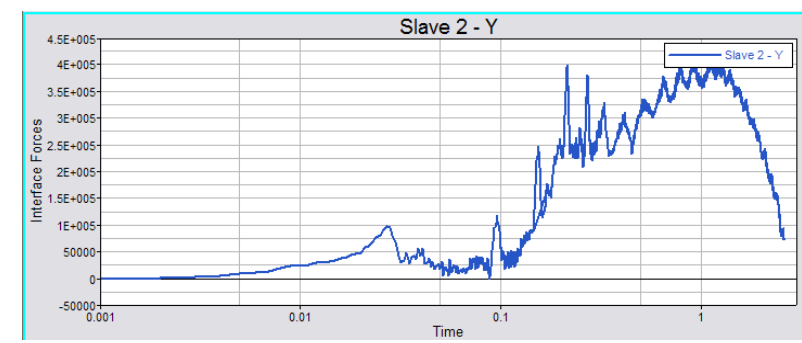

Fig.2 Axial Spinning Force

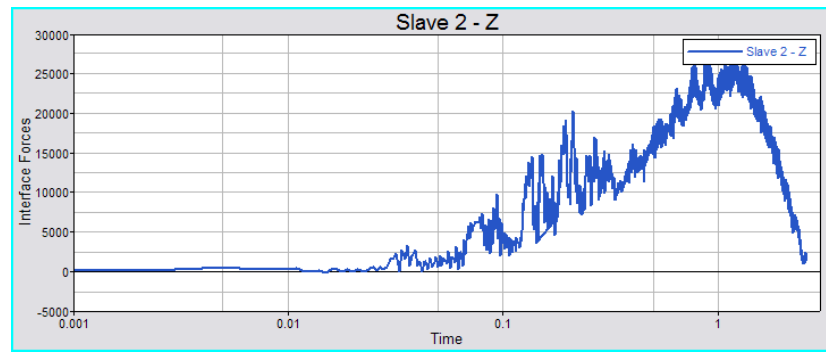

Fig.3 Shear Spinning Force

\section{Spinning Process Experiment}

The XYQK20-500 double spinning roller type of numerical control spinning machine from certain research institution is utilized to carry out three kinds spinning force process experiments on the projectile body of the same model, in which the spinning forces involved are obtained from three different ways as follows: the spinning force of fixed value, the spinning forces by traditional algorithm and the spinning forces by new algorithm. Except for the spinning force, the rest of parameters of spinning process are the same with that listed in Table 2.

Tab.2 Experimental Results

\begin{tabular}{|c|c|c|}
\hline & Precision & Harshness \\
\hline Fixed value & $0.31 \mathrm{~mm}$ & 6.3 \\
\hline Traditional algorithm & $0.25 \mathrm{~mm}$ & 6.3 \\
\hline New algorithm & $0.16 \mathrm{~mm}$ & 3.2 \\
\hline
\end{tabular}

\section{Conclusion}

This paper has put forward a rolling force based algorithm to calculate the spinning force and the Electrometric Method is utilized to verify the accuracy of the algorithm; after being verified to be accurate, the algorithm is applied into the simulation experiment of power spinning on projectile bodies in which the double spinning rollers and varying spinning forces are adopted; it is verified from the spinning process experiment that the method can effectively improve the dimensional accuracy of the diameter of projectile body and lower its surface roughness. 


\section{References}

[1] Zhao Yunhao, Li Yanli. Technoligy and application of spinning. Beijing, 2008, Machinery Industry Press.

[2] Matsunok. Recent Research and Development in Metal Forming in Japan [J]. Mater, Process, Technol, 3(1997)51-54.

[3] Anon. Metal spinning in the auto motive industry [J]. Sheet Metal Industries, 18(1995)23-25.

[4] Xue Kemin, Wang Zhen, Lu Yan. Elasto-plastic FEM Analysis and Experimental Study of Diametral Growth in Tube Spinning [J]. Mater, Process, Technol, 175(1997)69-72.

[5] WANG Z T, XIE S S, JIN Q J. Elasto-plastic finite element analysis of hydrostatic extrusion with various mathematically contoured dies [J]. Proceedings of the 24th International Machine Tool Design and Research Conference, eds, Davies BJ, 58(1983)51-58.

[6] HAYAMA, Hiroaki KUDO. Experimental Study of Tube Spinning [J]. Bulletion of JSME. 775(1979)167-170. 